Rhode Island College

Digital Commons @ RIC

\title{
The Impact of a Focused Training on Advanced Practice Nursing Student's Experience with Communicating Difficult News
}

Nicholas A. Gernt

Follow this and additional works at: https://digitalcommons.ric.edu/etd

Part of the Other Nursing Commons

\section{Recommended Citation}

Gernt, Nicholas A., "The Impact of a Focused Training on Advanced Practice Nursing Student's Experience with Communicating Difficult News" (2018). Master's Theses, Dissertations, Graduate Research and Major Papers Overview. 266.

https://digitalcommons.ric.edu/etd/266

This Major Paper is brought to you for free and open access by the Master's Theses, Dissertations, Graduate Research and Major Papers at Digital Commons @ RIC. It has been accepted for inclusion in Master's Theses, Dissertations, Graduate Research and Major Papers Overview by an authorized administrator of Digital Commons @ RIC. For more information, please contact digitalcommons@ric.edu. 
The Impact of a Focused Training on Advanced Practice Nursing Student's Experience with Communicating Difficult News

by

Nicholas A. Gernt

A Major Paper Submitted in Partial Fulfillment

of the Requirements for the Degree of

Master of Science in Nursing

in

The School of Nursing

Rhode Island College

2018 


\begin{abstract}
Healthcare providers often have the difficult job of communicating difficult news to patients and patient families. However, few practitioners report having formal instruction on a systematic method for the delivery of difficult news. The purpose of the program was to give a cohort of Advanced Practice Registered Nurses (APRN) students in their final semester of their graduate program formal instruction on how to effectively deliver difficult news to their patients and patient families. The role of the APRN is ever expanding and APRNs will often find themselves treating more complex patients in their practice. Being prepared for an inevitable encounter with a poor diagnosis or the death of a loved one is an invaluable tool that has proven to enhance patient and provider relationships and alleviate provider frustration. The program incorporated formal instruction that included a lecture on evidence based theory using the SPIKES method of the delivery of difficult news as the backbone for the teaching and reference. Students participated in triads, role-playing the SPIKES method following the lecture. A sample comprised of 11 students representing $58 \%$ of the class met the inclusion criteria. The program was evaluated using a researcher designed and validated Likert type survey, measuring learner confidence both pre- and post-instruction. Confidence levels were measured and all who responded to the post-instruction survey reported increased confidence $(>90 \%)$ in the delivery of difficult news.
\end{abstract}




\section{Table of Contents}

Background/Statement of the Problem ................................................................. 1

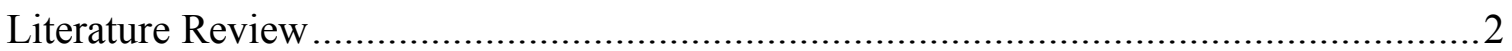

Theoretical Framework .................................................................................. 14

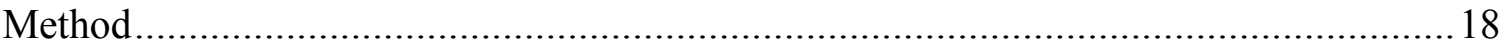

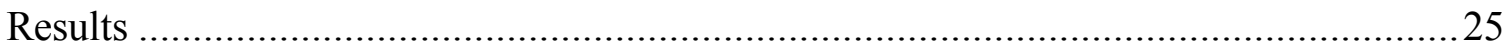

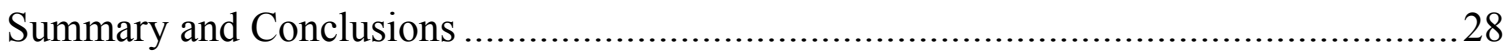

Recommendations and Implications for Advanced Nursing Practice.......................... 30

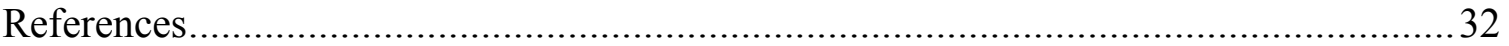

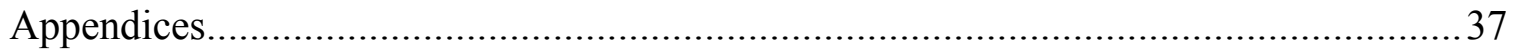


The Impact of a Focused Training on Advanced Practice Nursing Student's Experience with Communicating Difficult News

\section{Background/Statement of the Problem}

Health care providers often have the difficult job of communicating difficult news to their patients, families, and caregivers. Difficult news can range from an unexpected complication, need for additional time in a hospital, a new or upsetting diagnosis, or the death of a loved one. Although the delivery of difficult or bad news is a near daily occurrence in the healthcare setting, very few practitioners reported receiving formal training on the topic (Alelwani and Ahmed, 2014). In a review of the literature, very few clinical trials address the topic of communicating bad news and fewer being randomized controlled trials. The lack of research highlights a clear gap in the literature regarding the training and preparation of future practitioners to lead difficult conversations with patients and families. For example, according to the 2016 Adult-Gerontology Acute Care and Primary Care Nurse Practitioner Competencies (Thomas, et al., 2016), nurse practitioner (NP) students are not explicitly required to be trained in the delivery of difficult news. It is recommended that anticipatory guidance and counseling for individuals and their families based on identified health promotion needs, social support, and health status be taught; however, it is only suggested content, not required. This is a gap in current APRN education regarding specific training on how to deliver difficult or bad news.

The purpose of this program was to develop an informational program that prepares the learner to identify a need and utilize a structured framework to deliver difficult news. The program took place at Rhode Island College, in the MSN Program, Adult-Gerontology Acute Care Program, during a NURS 620 Skills Development Day. 
The students were offered education and training using tools necessary to effectively deliver bad news to patients and families using the SPIKES protocol.

\section{Literature Review}

A thorough literature review was conducted utilizing the CINAHL, PubMed, and OVID databases to search for articles published between 2006 and 2017 using the keywords nurse practitioner, bad news, delivering news, communication, simulation, end of life, and SPIKES. A small amount of literature related to these topics was found. All articles retrieved were in English only.

\section{Defining Difficult News}

Inevitably, within healthcare settings, undesirable health outcomes will exist. Patients and families need to be told of catastrophic diagnoses up to and including death. Some experts refer to this process as delivering difficult news (Barclay and Maher, 2010). Other experts call this news "bad news" and recognize that a multidisciplinary team approach is often used in the delivery of such news (Davenport and Schopp, 2011). It has been said that some of the most difficult communication with patients and families revolves around transitioning goals of care from curative to palliative goals (Wyckoff \& Houghton, 2009). What makes this news "difficult" or "bad" is the general premise that health care providers have compassion, which is defined as "deep awareness of the pain and suffering of others" (Venes, 2001 p. 459). It is compassion that allows those communicating difficult news to do so in a way that can ease the suffering of those receiving the difficult news. 


\section{Impact of the Delivery of Difficult News}

When delivered in an insensitive and untrained manner, the reception of difficult news can lead to increased distress, anger, and risk of litigation by the recipient while contributing to increased stress for the deliverer (Fallowfield and Jenkins, 2004).

Fallowfield and Jenkins go on to explore the notion that inadequate training in the delivery of bad news leads to ineffective delivery of such news and providers are left to deal with the resulting emotional sequelae. Buckman (1984) suggested reasons why communicating difficult news is challenging. The author identified lack of training, fear of blame, fear of not having all of the answers, and fear of expressing emotion as some factors that make communicating difficult news, difficult. These obstacles remain as challenges in today's complex healthcare delivery system.

\section{Ethical and Legal Considerations in Communicating Difficult News}

According to the American Nurses Association Code of Ethics, the guiding principle behind all nursing practice is a commitment to the right to self-determination (American Nurses Association, 2015). A key component includes the need to provide patients and their families with as much information as they want about their medical condition, which in turn allows patients to actively participate in an informed manner, in their care. The patient decides what treatment they will and will not receive, based on the honest and informative communication provided by their providers.

Legally, it has been suggested that many malpractice claims could be avoided if better communication between providers and patients occurs (Virshup, Oppenberg \& Coleman, 1999). The theory proposes that when patients are angry, they sue for 
malpractice as a mechanism to act out their anger. The authors discuss how risk management programs recognized this and developed training programs in communication to attempt to mitigate this risk. The authors further suggest that providers who are trained in communication and skilled in recognizing and responding to their patient's emotional needs are less likely to have litigation against them.

\section{Methods for Communicating Difficult News}

Much of the literature around communicating bad news comes from oncologic medicine. Research conducted between 1950 and 1970 showed that many practitioners in the oncology setting often failed to disclose important information to the patient or families when the outlook was unfavorable (Baile et al., 2000). The reasoning behind this decision was that it was considered inhumane to deliver unfavorable news regarding a poor prognosis. As time and oncology treatment options progressed, so did the need for a systematic approach to communicating difficult news. During a symposium in 1998, nearly 500 oncology practitioners took part in an interactive survey that revealed a high percentage of the providers $(>50 \%)$ delivered difficult news to patients on a regular basis. This lead to a further discussion that identified a gap in a common delivery method for discussing difficult news. Oftentimes, information was misinterpreted or misunderstood which leads to distrust and anger towards the practitioners and unrealistic expectations from the patient and families in regard to the prognosis of the disease (Baile et al., 2000).

When healthcare providers are educated on how to deliver difficult news, there are three methods that are commonly taught: The SPIKES protocol, the BREAKS method, and the $\mathrm{ABCDE}$ protocol. 
The SPIKES protocol is a six-step protocol which was developed by Baile et al. (2000) to address the need for a standardized method for communicating difficult news to the oncology patient. It consists of the following components; setting up the interview, assessing the patient's perception, obtaining the patient's invitation, giving knowledge, addressing the patient's emotion with an empathetic response, strategy, and summary. The first step in this process is $\mathrm{S}-\mathrm{SETTING}$ up the interview. This step includes such processes as arranging for privacy, involving significant others, sitting down, making a connection with the patient/family, and managing time constraints and interruptions. The second step in this process is $\mathrm{P}$ - assessing the patient's PERCEPTION. During this step, the medical provider uses open-ended questions to assess the patient or family's perception of the medical situation. The third step in this process is I - obtain the patient or family's INVITATION. The majority of patients and families express the desire for full information disclosure in regard to medical care, but not all patients desire this. The provider should inquire how much information the patient or family want to know about the given situation. The fourth step in this process is $\mathrm{K}$ - giving KNOWLEDGE and information to the patient/family. By warning a patient/family that bad news is coming, the provider may be able to lessen the shock that ensues after the disclosure of bad news. The fifth step in this process is E - addressing the patient/family's EMOTIONS with empathic responses. Assessing and appropriately responding to the patient's emotion is a difficult skill to master, but an important one nonetheless. The sixth step in this process is S - STRATEGY and SUMMARY. Patient/families who have a clear plan for the future are less likely to feel anxious and unsettled. When systematically combined and utilized effectively, the SPIKES method has proved to be an effective and appropriate 
vehicle for the delivery of bad news in most medical situations that requires a sensitive and understanding approach (Baile et al., 2000).

A survey sent out by Baile to oncologists who were trained in using the SPIKES protocol revealed that the respondents (99\%) found the method practical and easy to use. However, over half of the respondents identified difficulty in using empathetic, validating, and exploring statements when responding to patient's emotions as a limiting factor in effectively using this protocol. Baile et al. (2000) also highlighted that there was still no specific outlined training for the delivery of bad news in medical undergraduate and post-graduate programs. In fact, most oncologists reported learning how to deliver difficult news by observing more senior practitioners in their practice settings (Baile et al, 2000).

The BREAKS method is a second method developed for communicating bad news (Narayanan, Basta, \& Koshy, 2010). The BREAKS method consists of the following steps; background, rapport, exploring, announcing, kindling, and summarize. In the first step, the medical professional must educate him or herself on the full details of the news to be communicated. This means developing the most comprehensive understanding of the diagnosis and prognosis in order to be able to effectively answer any questions the patient or family may have. Additionally, considering the patient's background and their cultural or ethical considerations prior to the conversation is important. Next, in developing rapport, the provider must take care to avoid patronizing the patient or family and also aid the patient and family in feeling more prepared to receive the news. The provider then explores the different levels of understanding of the patient and the patient's family of the condition. The provider must be careful to not 
provide premature reassurance before thoroughly exploring and understanding the concerns. During this time, the provider should outline facts and prognosis details, but certainties of longevity should not be given to the patient. After developing rapport, the provider must state the difficult news. In this step, the provider is encouraged to mirror the recipient's body language, avoid medical jargon, and use caution with utilizing euphemisms. During the kindling step, the provider answers any questions or clarifies any aspect of the information that was unclear. It is during this step that the provider should attempt to ascertain if the patient and family understood the difficult news which was delivered. Finally, the provider summarizes the information delivered and arranges for follow up and plans following the delivery of the bad news, keeping in mind that very difficult news may leave the recipient unstable emotionally.

The BREAKS method highlights the fact the bad news is simply bad news, no matter how well it is said. The authors suggest that ineffective communication of difficult news creates a chasm in the patient and provider relationship. This, in turn, may lead to a divide and eventual avoidance by the patient for future care. The authors also suggest that provider education and training in communicating difficult news is important and valuable. Specifically, they suggest role play can be useful in developing better communication skills for delivering difficult news.

Finally, the ABCDE protocol developed by Rabow and McPhee (1999) involves advanced preparation, building a therapeutic relationship, communicating well, dealing with patient and family reactions, and encouraging and validating emotions. This method brings forth the notion that breaking bad news is not a delivery, but a dialogue between two people who are trying to understand a meaning much greater than themselves. This 
model delves more into the spiritual side of the delivery of bad news and assumes a certain amount of understanding on the part of the patient that in the end, we are simply destined to perish. The author takes on a more humanistic approach in highlighting that the provider must understand that faith in the process of life and death will help guide the dialogue to an agreed upon understanding of the necessity and eventual death that occurs with all life.

An expert opinion written by Rosenzweig (2012), an Advanced Practice Oncology Nurse Practitioner highlights the need for a standardized method of teaching practitioners how to deal with difficult situations in regard to communication. The author suggests that using the SPIKES protocol eliminates the shortcomings of other methods and improves the experience for both the deliverer and receiver of the bad news. Rosenzweig goes on to say that without excellent, empathetic communication skills, the provider is not fully able to relate with their patient and patients' families in a way that will solidify a trustworthy and fruitful relationship. This article is an expert opinion and has data to support effectiveness, however, the author believes the SPIKES protocol may prove to be highly effective if implemented as part of a standardized teaching plan for NP and NP students. Based on the systematic approach that includes an interpretation and evaluation phase, Rosenzweig recommends that the SPIKES protocol provides the most straightforward and effective delivery method to use during a program development. Rosenzweig states that NP students should be exposed to the techniques in the SPIKES protocol in a physical assessment or differential diagnosis course first as a didactic phase, then later used in practice during a controlled clinical phase to solidify learned knowledge with practical application. Rosenzweig suggests this would be the most 
effective way of introducing and incorporating communication techniques into the NP curriculum.

In 2016, Cory and Gwyn published a qualitative research study which suggested that using the SPIKES protocol to educate NP had the potential to make the difficult job of communicating bad news better for the provider. However, the study was limited to five experienced NPs working on a single oncology unit communicating the bad news of a difficult cancer diagnosis over the period of one month. This study did not address how to deliver bad news to a family when the patient is not participatory in the conversation and it only explored the experiences of experienced practitioners, not novice practitioners. The author believes that education on this protocol with new oncology NP's has the potential to affect the NP and patients positively and may have a lasting impact on their relationship and future encounters.

\section{Provider Training on Communicating Difficult News}

A perfect framework does not exist in regard to communication in general (PopaVelea \& Purcărea, 2014). In healthcare, there are often times when a provider will be faced with a unique interaction between a patient or patients' family that the provider is not prepared to deal with. By giving the provider a guiding framework, it allows the provider to have a more structured basis from which to deliver difficult news to either a patient or patients' family member. The authors further suggest that formalized training in communication can lead to enhanced patient satisfaction and has the capability to improve outcomes through improving adherence, as well as improving patient satisfaction. 
In a 2015 literature review, Lamba, Tyrie, Bryczkowski, and Nagurka, identified a gap in teaching and assessing communication skills in regard to communicating with families following an unexpected death. The study analyzed data from 120 medical students who completed a pre- and post-clerkship survey. The authors suggested that surgery residents may benefit from using simulation to support them when communicating bad news. Similarly, Lamba (2015) suggested that teaching emergency medicine students how to deliver bad news using a structured communication module, practiced via simulated resuscitation scenarios lead to self-reported increased knowledge, comfort, and competence in communicating bad news. Simulated scenarios lead the students to experience strong emotional responses; these responses should be discussed in training, in order to allow students to better handle them in a way that is emotionally healthy (Curtis et al., 2013).

A 2011 review of literature (Reid \& McDowell) suggested best practice guidelines for communicating bad news. In this double-blind, peer-reviewed literature review, the authors reviewed literature from the last 30 years to determine what form the best practice of communicating bad news should take. The authors reviewed a total of 22 articles and guidelines published between 1981 and 2009. These articles included various communication skills, difficulties with breaking bad news, and challenges with end of life discussions. The authors determined that there are certain principles which should guide the communication of bad news in the acute care setting, including using clear language and supporting the bereaved regardless of their reaction to the news. In this review of literature, the bereaved often felt that they were informed of bad news in a poor and uncaring way. The perceived lack of training in communicating bad news had a 
detrimental effect on grieving and made the impact of the death of loved ones more devastating for the bereaved. The article suggested using a six-step protocol for communicating bad news. However, the authors cautioned against relying too heavily on a prescribed script. In their review of the literature, they found the bereaved preferred hearing bad news from police rather than from medical professionals as the police were 'freer with their emotions'. The perceived empathy the police demonstrated when communicating bad news was felt to be more real and more human than when medical professionals were communicating in a way that felt pre-planned and generic. The authors suggest that there is a need for more focused and specific training for all healthcare personnel in the area of communicating bad news. The authors also suggest that having excellent communication skills with particular attention paid to both verbal and non-verbal communication, as well as insight into the provider's own bias has the potential to alleviate some stress felt by the bereaved.

A recent study published by Bays et al. (2014) addressed the concern of not being adequately prepared to deliver such news. Their research evaluated the effectiveness and change (if any) of the learner communicating bad news and expressing verbal empathy after receiving a standardized training. The study used internal medicine subspecialty fellows, as well as NP students from the University of Washington and the Medical University of South Carolina. The quantitative, quasi-experimental design had the fellows participate in a simulation training called "Codetalk". Before and after the simulation, the participants were given self-evaluations measuring their confidence levels while interacting with the standardized patients. Of the 145 participants enrolled, their scores improved from a mean total score of 8 to a mean score of $11(p<0.05)$ in a coded 
behavior grading scale. Factors that limited this study included the lack of association between any significant improvements and any discernable participant characteristics. Also, there appeared to be a disproportionate representation of physicians to NPs ( $52 \%$ physician, $14 \% \mathrm{NP})$. The control group in this study were fellows unable to enroll in the program.

Similarly, a study performed by Rosenzweig et al. (2008) addressed the needs of NP students in simulation training. Their research study focused on using a simulation lab skills workshop designed to increase the NP's comfort level and perceived ability to initiate difficult conversations. This study involved 38 NP students from an Acute Care Nurse Practitioner program at a major university. The study, developed in collaboration with the schools of nursing and medicine, had students attend a didactic session, as well as a 2-hour simulation concentrated on breaking bad news, empathetic communication, motivational interviewing, and communicating with the "angry" patient. The study was conducted in a quantitative, quasi-experimental fashion with the data collection via survey. The findings were based on two focus areas, both of which showed improvement. Students reported confidence in communicating news showed a marked improvement on a confidence scale of 1-7 from a mean score of 4.4 pre-training, to a mean score of 5.6 post training $(p<0.001)$. This study also included a 4-month followup as a measure of long-term retention with scores dropping slightly to 5.3. Students ability to initiate difficult conversations scale of 1-7, also saw similar score improvements with the pre-training mean scores of 4.2 increasing to 5.7 after the training and the 4-month follow-up mean score of $5.2(p=0.001)$. Limiting factors for this study 
was the relatively small sample group with reliance on self-reporting and the complexity of scenarios that were given to the students, which made consistent replication difficult.

The Competencies for Nurse Practitioners (Thomas et al, 2017) are the most current competencies from which The National Organization of Nurse Practitioner Faculties bases the core competencies for all nurse practitioners in educational programs. A careful review of the competencies reveals no explicit competency in regard to communication in difficult situations. This highlights a lack of need for the nurse practitioner to be competent in this skill.

After careful review of the literature surrounding the concept of communication of bad news, a few common elements stand out. First and foremost, patients and their families do not always feel that bad news is communicated in a manner that is empathetic (Sangeeta, Tyrie, Bryczkowski \& Nagurka, 2016). Training around communicating bad news is limited in healthcare professional programs including Advanced Practice Registered Nurse (APRN) programs. The preferred method to communicate bad news is using a systematic approach which can be taught and refined over time. Throughout the literature, the SPIKES protocol is identified as a favorable method by which to communicate bad news. Research also suggests that after a training on how to best communicate bad news, learners report an increased confidence in their ability to communicate bad news in an effective manner. By developing a training program, the APRN students learn how to communicate bad news with empathy. They also develop the ability to communicate bad news in a systematic approach that allows for clear and open communication. As providers, APRNs are more and more often tasked with this daunting challenge. 


\section{Theoretical Framework}

This project was guided by two theoretical frameworks, Knowles Adult Learning Theory as well as the Logic Model. Knowles theory addressed the importance of designing the education focusing on the unique needs of adult learners. The Logic Model provides an organizing framework for the development and evaluation of the learning module.

\section{Adult Learning Theory}

The theoretical framework used to guide this project is Knowles 'Adult Learning Theory (Knowles, Holton, \& Swanson, 2005). The framework focuses entirely on the adult as the learner and the unique needs of adults in an academic setting. Knowles theorized that the single most important thing in helping adults learn is to create a climate of physical comfort, mutual trust and respect, openness, and acceptance of difference (Knowles, Holton, \& Swanson, 2005). Knowles established that adults have very specific needs in regard to learning. The specific unified theory for adult learning is called andragogy. Andragogy differs from pedagogy (youth learning) in that the focus lies in the teacher utilizing the life-experience of the adult to facilitate learning as opposed to simply teaching content instead of processes. Knowles proposed a seven-step teaching process that include: creating a cooperative learning environment; planning goals mutually; diagnosing learner needs and interests; helping learner to formulate learning objectives based on their needs and individual interests; designing sequential activities to achieve these objectives; carrying out the design to meet objectives with selected methods, materials, and resources; and evaluating the quality of the learning 
experience for the learner that includes reassessing needs for continued learning (Blondy, 2007).

Knowles' Adult Learning Theory highlights a set of complex variables due to the fact that no adult has the same life-experience and therefore may not synthesize information in the same way as another adult learner. Knowles' Assumptions of Adult Learners guides the teacher to allow for such variances within the teaching process to make each teaching scenario as effective as possible, which in turn leads to a higher percentage of content learned. Adult learners require an individualistic approach to teaching and learning. By following Knowles' basic theory for teaching adults, the teacher is able to individualize and effectively teach the content to each student in the most effective manner possible. The instructor needs to discuss with participants their experiences with communicating bad news and then incorporate participants' experiences into the scenarios based on common themes. This can be done by forming a standardized set of two scenarios designed for individuals who have had little to no experience, and some with prior experience with the delivery of bad news. This will both enhance the value of the experience for all participants and create the most realistic scenarios possible. Grounding the project in Knowles theory will create a learning experience that is valuable to the adult participants who often come to learn from previous experiences that are highly valuable to the learning process.

\section{The Logic Model}

The second theoretical framework that guided this project is the Logic Model developed by the W.J. Kellogg Foundation (2004). The model focusses on a systematic and visual way to view the relationship between available resources, planned activities, 
and anticipated results. The model accomplishes these tasks by utilizing a 5-step process that includes monitoring available resources and inputs, overseeing the activities and outputs, measuring the outcomes, and evaluating the overall impact the project has made and adjusting teaching or expectations as needed (W.K. Kellogg Foundation Logic Model Development Guide, 2004). This type of model is a fluid model that allows the educator to systematically monitor each step of the teaching phase so that resources, activities, and outcomes can be measured and adjusted to achieve the greatest success. By utilizing the steps of the Logic Model, the developer is able to use a guide from which to develop the majority of the teaching program. The developer uses data gathered from the postteaching survey in order to evaluate the program based on the guiding principles of the Logic Model. By following this model, the educator develops a teaching program that is highly effective and responsive to the learners.

The first two steps of the Logic Model include the Inputs/Resources, as well as Activities which is the work planned for the project. The inputs and resources for this project were limited financially and limited in regard to participants' availability and time allotted. This is due to the time allocation for other required teachings in the graduate program, as well as the limited number of students enrolled in the graduate program at any one time. The activities involved in this program included a pre-test, lecture, roleplaying, post-test, and data collection that measured the effectiveness of the program and allowed for adjustment and refinements prior to future curricular designs.

The last three steps of the Logic Model include the Outputs, Outcomes, and Impact steps and are part of the intended results of the project. The outputs are the direct products from the program, and in this case, a better understanding of how to effectively 
delivery of difficult news and recognize the profound impact on the patient and family experience during a challenging healthcare situation. The outcomes are the measurable impacts that are measured from the teaching and were collected during the pre-test and post-test portion of the program and the data collected will guide future teaching. The impact of this teaching was to bring a higher level of awareness of the importance of effectively communicating difficult news to APRN students enrolled in a master's degree program. 


\section{Method}

\section{Purpose and Question}

The purpose of this program was to develop an informational program that prepares the learner to identify a need and utilize a structured framework to deliver difficult news. The program provided APRN students with the tools necessary to effectively deliver bad news using the SPIKES protocol.

\section{Design}

The program consisted of three parts. The first part of the program was to conduct a survey of APRN student's prior to introducing the program. This survey was distributed via email in order to allow participants to take the time they need to complete the survey. The program developer created a 6-question survey (Appendix A) which was piloted by three APRN student peers other than potential program participants for their review. The survey included questions asking for the level of confidence and previous experience in communicating bad or unfavorable news to a patient or family member of a patient. The responses used a Likert scale in order to extract quantitative data.

The second aspect of the program included the implementation of a teaching module, teaching participants how to deliver bad news utilizing the SPIKES protocol. This was the most time intensive component of the program. The first part of the module was a power point presentation which reviewed the goals and objectives of the program and then the SPIKES protocol was explained, including how and when to implement it in the clinical setting. The presentation culminated with a video taken from the Institute for Healthcare Improvement (IHI) that showed examples of proper and improper communication techniques, allowing the APRN's to see what examples of each looked like from an observer's perspective (Prose \& Haglund, 2015). Following the 
presentation, the participants had the opportunity to participate in predetermined peer triads in order to practice using the SPIKES protocol to deliver difficult news with author developed scenarios. Each student was able to rotate through playing the role of the provider, the recipient of the difficult news, and as an observer. A discussion session was conducted after the role-playing experience to explore and share their personal experience with the exercises.

Finally, the post-intervention survey was administered with a 1-5 Likert Scale to extrapolate quantitative data following the teaching module and role-playing session. The data was evaluated for statistical significance to determine if teaching and practicing the SPIKES protocol improved participants' knowledge and confidence in the area of communicating bad news. The program was approved by the RIC Internal Review Board and approval was obtained from the course faculty teaching NUR 620 Skills Development Day.

\section{Sample}

The sample included all APRN students attending the NUR 620 Skills Development Day as a requirement of Nursing 620, scheduled on February 8, 2018. All students were invited to participate in the program. The sample size was 18 APRN students.

Site

The primary site for this project was located in a simulation classroom large enough to accommodate teaching, role-playing, and small group discussion in the Rhode Island Nursing Education Center (RINEC) located at 360 Eddy Street in Providence, RI. 


\section{Procedures}

The student surveys and teaching module were designed and led by the program developer and all information was gathered and synthesized in accordance with basic ethics and privacy for the students. No identifying data was obtained including any personal identifying information or other identifiable data on the pre-test and post-test surveys. The time requested for the program was 90-minutes, including a lecture piece on communicating bad news, a practice experience incorporating the role-playing of communicating bad news, and a brief discussion session providing time for questions or concerns related to the content and experience.

A 15-minute teaching piece was conducted at the beginning of the skills session which had the following desired outcomes:

1. Recognize the importance of effectively communicating bad news to patients and families

2. Understand the SPIKES protocol for communicating bad news

3. Apply newly learned skill in a safe, academic environment with a focus on growth and development

4. Analyze pre-existing deficits and barriers in personal communication style

5. Evaluate personal growth and increase the comfort level in communicating bad news Following the teaching portion of the program, a 45-minute role-playing exercise took place. APRN students were randomly placed in peer triads. Each student had one opportunity to communicate bad news, one opportunity to receive bad news, and one opportunity to record the interaction and provide feedback according to a SPIKES tip sheet (Appendix E) provided by the program developer. Each student participated in a 
fifteen-minute patient scenario with the patient outcome being either a difficult diagnosis or the death of the patient. Each participant was allowed five minutes during the fifteenminute scenario to provide feedback to their partner on their perception of the communication of the bad news. The group then reconvened and had an opportunity to participate in a ten-minute sharing, discussion, and feedback session. Once the exercise concluded, students were sent an email with a link to the post-survey. Following the same principles as the pre-survey, the post-survey did not collect any personal data.

\section{Measurement}

Pre-and post-survey data was collected using a Likert scale for the purpose of data collection and analysis. The pre-survey (Appendix A) contained six questions and the post-survey included nine questions (Appendix B), both developed by the program developer and previously tested by three peers. Questions 1-6 used a Likert scale format ranging from 'strongly agree' to 'strongly disagree', and questions 7-9 on the post-survey were 'yes' or 'no' answers. The post-survey mirrored the pre-survey for continuity of data collection and analysis with the exception of questions seven, and eight which are yes/no questions, and question nine which allowed for free-text by the participants.

\section{Timeframe}

The program was initiated with an emailed notification (Appendix C) of the upcoming program with a request to complete the pre-survey prior to the skills day in February 2018. The survey was designed to be completed in no more than 10-minutes. The teaching and skill development piece was conducted during NURS 620 Skills Day in February 2018, taking 90-minutes to complete, followed by the emailed post-survey (Appendix D) which took less than 10 minutes to complete. The data was collected and 
analyzed for statistical significance throughout the spring of 2018 and findings were presented at the MSN poster presentation at the Rhode Island College Eighth Annual Graduate Student Symposium in May 2018.

\section{Organizational/Systems Factors}

The resources needed for the pre-survey included a computer with internet, email access to students in NURS 620, and informed consent by participants. Barriers to completing the pre-survey included, but were not limited to equipment failure, power failure, website failure, unclear directions, and an opportunity to decline participation. Coordination with the NUR 620 faculty took place in the Fall of 2017 prior to the implementation of the program. The teaching program required 90 minutes of time from the NUR 620 Skills Day. Course faculty had to approve this time allotment. The program required access to the simulation lab for the conduction of the practice sessions. Barriers to conducting the on-site teaching included but were not limited to: equipment failure, power failure, inclement weather, non-participation, and approval from both the NURS 620 course faculty and the Rhode Island College Graduate School of Nursing Dean. The post-survey data collection required a computer with internet access. Support was provided by the Nursing Graduate faculty. Data collection was provided by the website www.surveymonkey.com (2018) which was used for all distribution and collection of data during both the pre, and post-survey.

\section{Desired outcomes}

The desired outcomes for this program were to design a teaching and practice session on the communication of difficult news for APRN students at Rhode Island 
College (RIC) and determine if the program increased participants knowledge and confidence in delivering difficult news.

\section{Ethical Considerations}

The sample consisted of students, who are viewed as vulnerable. However, the students who participated were not identified by any personal information on their surveys. The student surveys and teaching module were conducted by the project developer and all information was collected in accordance with basic ethics and privacy standards. The program developer is a peer, and not responsible for any rating of academic performance of any students. All NURS 620 students were invited to participate, however, four students chose not to participate with no ill consequences. The program developer took great care to address any cultural, spiritual, or other ethical considerations that may occur during the teaching module. End of life discussions can bring up sensitive matters for the participants, and the program developer remained astute to this possibility. If students demonstrated visual signs of distress, the Rhode Island College Nursing course faculty were present. The RIC Counseling Center was available and staffed with trained mental health professionals who can address the specific needs of those requesting assistance. The pre-survey was distributed with a cover letter addressing these ethical considerations and provided participants with contact information for support resources, as well as information as to how to decline to participate in the program (See Appendix A).

\section{Evaluation Plan}

The program was evaluated on a non-biased basis. All data from pre-intervention and post-intervention surveys was collected and analyzed. The program developer 
determined if the program was effective in improving the confidence of participants in the delivery of difficult news to patients and families by comparing the pre-and postintervention survey results. The developer identified areas of the program to improve based on the feedback students provided in the open-ended question portion of the posttest survey. All open-ended questions were analyzed for predominant themes and reported with the survey findings.

\section{Plans for Dissemination of Results}

Results of the effectiveness of the program will be presented as part of the developer's major paper for the RIC Masters' of Science in Nursing (MSN) program and available on Digital Commons through the RIC Library. Additionally, the project will be presented at the RIC Eighth Annual Graduate Student Symposium MSN poster presentation in the spring of 2018. 


\section{Results}

Of the 18 possible subjects, 15 subjects completed the pre-survey, representing $83 \%$ of the students meeting the inclusion criteria. The 15 students consisted of an unknown ratio of males to female's due to the anonymity of the online survey. All 18 students participated in the program. The post-survey was completed by 11 students in all representing $61 \%$ of eligible students.

Responses to the six Likert Scale questions demonstrated increased score from the pre-intervention to the post-intervention survey. When asked if the participants had confidence in their ability to effectively communicate difficult news to a patient, $46.6 \%$ “agreed", 26.6\% were "undecided", 13.3\% “disagreed", and 13.3\% "strongly disagreed" during the pre-intervention survey. Data collected from the post-intervention survey revealed $36.3 \%$ participants "strongly agree", and $63.6 \%$ of participants "agree" that their confidence in the ability to effectively deliver difficult news to patients. Question three revealed, pre-intervention, that nearly $60 \%$ of the participants "strongly disagree" or "disagree" when asked if they had a standard method for communicating difficult news to patients and patient families. Post-intervention, the same question was asked and over $90 \%$ of the respondents answered with an "agree" or "strongly agree" response. Table 1 illustrates questions, the mean response pre, and post-intervention total mean scores, as well as the overall change following the program. 
Table 1.

Likert Scale Confidence Pre-and Post-Test Survey Results

\begin{tabular}{|c|c|c|c|c|}
\hline & Survey Questions & $\begin{array}{c}\text { Pre- } \\
\text { Survey } \\
\text { Results } \\
\text { Mean } \\
\text { Score }\end{array}$ & $\begin{array}{l}\text { Post- } \\
\text { Survey } \\
\text { Results } \\
\text { Mean } \\
\text { Score }\end{array}$ & $\begin{array}{l}\text { Change } \\
\text { in } \\
\text { Score }\end{array}$ \\
\hline 1. & $\begin{array}{l}\text { I have confidence in my } \\
\text { ability to effectively } \\
\text { communicate difficult news } \\
\text { to patients. }\end{array}$ & 3.1 & 4.4 & +1.3 \\
\hline 2. & $\begin{array}{l}\text { I have confidence in my } \\
\text { ability to effectively } \\
\text { communicate difficult news } \\
\text { to patient families. }\end{array}$ & 3.2 & 4.4 & +1.2 \\
\hline 3. & $\begin{array}{l}\text { I have a standard method of } \\
\text { communicating difficult } \\
\text { news to patients and patient } \\
\text { families. }\end{array}$ & 2.3 & 4.5 & +2.2 \\
\hline 4. & $\begin{array}{l}\text { I am confident in my } \\
\text { understanding of the } \\
\text { barriers to communicating } \\
\text { difficult news to patients } \\
\text { and patient families. }\end{array}$ & 2.9 & 4.4 & +1.5 \\
\hline 5. & $\begin{array}{l}\text { I have had many } \\
\text { opportunities to effectively } \\
\text { communicate difficult news } \\
\text { to patients and patient } \\
\text { families. }\end{array}$ & 3.1 & 3.2 & +0.1 \\
\hline 6. & $\begin{array}{l}\text { I am interested in learning } \\
\text { an effective method for } \\
\text { communicating difficult } \\
\text { news to patients and patient } \\
\text { families. }\end{array}$ & 4.5 & 4.6 & +0.1 \\
\hline
\end{tabular}




\section{Program Evaluation}

There were two additional questions on the post-intervention survey that were not included in the pre-intervention survey which addressed how the learners felt the program effected their future practice. These questions included subjective views based on a simple yes/no answer for the purpose of evaluating the efficacy of the teaching and if the program goals were met. All subjects answered both questions "yes," indicating they believed the program gave a better understanding of how to communicate difficult news and that they believed the skills and knowledge learned in the program would help them in the future.

The respondents were asked to provide subjective comments, concerns, or other information in regard to the teaching they received as part of the post-survey. In all, a total of three comments were received out of the eleven respondents to the post-survey. Comments included: “Great learning!”, “Great education and very applicable”, and “Great Program, well presented, and well received." No overwhelming flaws or comments on future improvements were noted. 


\section{Summary and Conclusions}

Research shows that there is a need for an effective way to communicate difficult news to patients and patient families (Baile et al. 2000). The APRN is employed in a variety of settings that put them in a position to deliver difficult news to patients and families, therefore there is a need for education and training in the delivery of difficult news (Alelwani \& Ahmed, 2014). A review of the literature shows that though most APRNs will find themselves in a position to deliver difficult news, very few have been formally instructed on how to do so (Alelwani \& Ahmed, 2014).

The purpose of this program was to develop an informational program that prepares the learner to identify a need and utilize a structured framework to deliver difficult news. This program focused on the RIC NURS 620 APRN students during their final semester of the MSN program at RIC and facilitated by the NURS 620 faculty during the Skills Day in February 2018. The method of instruction included an authordeveloped power point presentation on the SPIKES method, a student role-playing training supervised by the student researcher and NURS620 faculty, and a debriefing that included a discussion, and question/answer session. Recruitment efforts resulted in all 18 students participating in the hands-on teaching portion of the project. A Likert type survey was utilized for both the pre-and post-test surveys to evaluate the effectiveness of the program. The survey measured the learner's knowledge and confidence both before and after the instructional period. The data collected revealed a positive response from all who participated in the survey, helping lend validity to the program. The data collected strongly suggested the program objectives and desired outcomes were met.

The program educated learners on the need for education in the delivery of

difficult news in the clinical setting. The program developer brought forth evidence-based 
methods for the delivery of difficult news, provided teaching in the area of delivering difficult news, and allowed learners to practice their newly acquired skills in a controlled and supervised setting. Discussion after the teaching program produced many questions that stimulated conversation and critical thinking. Some of the topics brought up during this discussion ranged from difficult scenarios and how to navigate them, as well as past experience and how having a standardized method for delivering difficult news could have been helpful.

The data gathered during the survey demonstrated that there was a great amount of interest in learning the skills necessary to effectively deliver difficult news to patients and patient families. The interest was evident in both the pre, and post-intervention survey. This high level of interest may have lent to the success of the program and supports the need for such programs in the future. The learners all agreed that the content taught would be beneficial in their future practice areas regardless of the setting. The program fulfilled graduate nursing student's educational needs that were otherwise not addressed in the course curriculum.

\section{Limitations}

There were a few limitations identified with this program. Time was something that proved to be a limiting factor during this project. As with learning any new skill, it is difficult to produce mastery of a subject with only limited exposure (Odhayani \& Ratnapalan, 2011). Successful learning and mastery of a communication skill are best found when subjects are able to utilize what they have learned, in a controlled environment and then observed and mentored in a practice environment by a peer proficient in effective communication skills and provide positive feedback. Another 
limiting factor was the relatively low number of participants enrolled in the program. A larger scale teaching workshop with a larger sample size may have resulted in different outcomes, therefore statistical significance cannot be determined at this time (Jakobsen, Gluud, Winkel, Lange, \& Wetterslev, 2014).

\section{Recommendations and Implications for Advanced Nursing Practice}

The overall success of an educational program should not be determined solely on the data that is collected (Fitzgerald et al., 2012). The real success is measured in the ability for the learners to successfully take the knowledge they have learned and effectively use it in practice. The participants in this program now have a basis of knowledge on how to effectively communicate difficult news to patients and patient families that they were once lacking. Multiple students have expressed gratitude for the teaching after having used the methods learned in their clinical environments since the program commenced. This is evidence of the need for this program and its practical application in future employment settings for these APRN students.

Nursing education, in particular, graduate schools of nursing, falls short on teaching effective methods of communicating difficult news to their students (Thomas et al, 2017). Evidence gathered during the pre-intervention survey revealed that very few, if any, of the students stated they had received a formal education in how to effectively deliver difficult news via a structured method such as the SPIKES method for delivering difficult news to patients and patient families. During the pre-intervention survey, the responses indicated that there was universally a high interest in learning an effective way of communicating difficult news to patients and patient families. 
The program developer encourages the RIC School of Nursing (SON) to continue utilizing this program in future graduate classes. Furthermore, the program developer recommends the RIC SON faculty include this content as part of the curriculum for the senior level graduate students as part of their final Skills Day so they develop a method for the delivery of difficult news to patients and patient families prior to graduating. This program was deemed a success by the students involved as evidenced by the survey data collected. A clear gap in knowledge was identified and a structured teaching program was developed and implemented. The student researcher identified small changes that could be made in future iterations of this program, but the overall content delivered and take away points remain the same. Future changes in the program include more hands-on time to role-play and practice, and a larger population of students involved in order to continue to collect data in order to improve the program. The program developer believes this is an important content area that should be included not only for the purpose of the student knowledge and comfort but also for the future recipients of difficult news. This is not limited to just the RIC SON, but may also be useful on a national level as a course content that should be a required content for all MSN programs. In fact, currently practicing APRNs should have access to a program such as the one developed for this project in order to grow in their practice. This program has the potential to improve how APRNs deliver difficult news to where it is done in a way that is effective and allows enhancement of the provider and patient relationship rather than causing undue strain and improve overall patient outcomes. 


\section{References}

Alelwani, S. M., \& Ahmed, Y. A. (2014). Medical training for communication of bad news: A literature review. Retrieved from https://www.ncbi.nlm.nih.gov/pmc/articles/PMC4113982/

American Nurses Association. (2015). Code of ethics for nurse with interpretive statements.

Baile, W. F., Buckmanb, R., Lenzia, R., Globera, G., \& And, E. A. (2000, August 01). SPIKES - A six-step protocol for delivering bad news: application to the patient with cancer. Retrieved from http://theoncologist.alphamedpress.org/content/5/4/302.full

Barclay, S., \& Maher, J. (2010). Having the difficult conversations about the end of life. British Medical Journal, 341, 4862-4869

Bays, A. M., Engelberg, R. A., Back, A. L., Ford, D. W., Downey, L., Shannon, S. E., ... Curtis, J. R. (2014). Interprofessional communication skills training for serious illness: Evaluation of a small group, simulated patient intervention [Research Article]. Journal of Palliative Medicine, 17(2), 159-66.

Blondy, L. (2016). Evaluation and application of andragogical assumptions to the adult online learning environment. Journal of Interactive Online Learning, 6(2), 116130. Doi:10.18411/d-2016-154

Buckman, R. (1984). Breaking bad news: Why is it still so difficult? British Medical Journal (Clinical Research Ed.), 288(6430), 1597-1599. 
Corey, V. R., \& Gwyn, P. G. (2016). Experiences of nurse practitioners in communicating bad news to cancer patients. Retrieved from http://www.advancedpractitioner.com/issues/volume-7,-number-5-(julaug2016)/experiences-of-nurse-practitioners-in-communicating-bad-news-to-cancerpatients.aspx

Curtis, J. R., Back, A. L., Ford, D. W., Downey, L., Shannon, S. E., Doorenbos, A. Z., ... Engelberg, R. A. (2013). Effect of communication skills training for residents and nurse practitioners on quality of communication with patients with serious illness: A randomized trial. JAMA, 310(21), 2271-2281. http://doi.org/10.1001/jama.2013.282081

Davenport, L., \& Schopp, G. (2011). Breaking bad news: Communication skills for difficult conversations. Journal of the American Academy of Physician Assistants,24(2), 46-50

Fallowfield, L., Jenkins, V. (2004). Communicating sad, bad, and difficult news in medicine. Lancet, 363 North American Edition (9405), 312-319.

Fitzgerald, C., Kantrowitz-Gordon, I., Katz, J., \& Hirsch, A. (2012). Advanced practice nursing education: Challenges and strategies. Retrieved from https://www.ncbi.nlm.nih.gov/pmc/articles/PMC3246751/

Jakobsen, J. C., Gluud, C., Winkel, P., Lange, T., \& Wetterslev, J. (2014). The thresholds for statistical and clinical significance - A five-step procedure for evaluation of intervention effects in randomized clinical trials. BMC Medical Research Methodology, 14, 34. http://doi.org/10.1186/1471-2288-14-34 
Knowles, M. S., Holton, E. F., \& Swanson, R. A. (2005). The adult learner: The definitive classic in adult education and human resource development. Amsterdam: Elsevier.

Lamba, S., Nagurka, R., Offin, M., \& Scott, S. R. (2015). Structured communication: Teaching delivery of difficult news with simulated resuscitations in an emergency medicine clerkship. Western Journal of Emergency Medicine, 16(2), 344-352. http://doi.org/10.5811/westjem.2015.1.24147

Narayanan, V., Bista, B., \& Koshy, C. (2010). 'BREAKS' protocol for breaking bad news. Retrieved from https://www.ncbi.nlm.nih.gov/pmc/articles/PMC3144432/

Odhayani, A., \& Ratnapalan, S. (2011). Teaching communication skills. Canadian Family Physician, 57, 1216-1218. https://www.ncbi.nih.gov/pmc/articles/PMC3192093/pdf/0571216.pdf

Popa-Velea, O., \& Purcărea, V. (2014). Issues of therapeutic communication relevant for improving quality of care. Retrieved from https://www.ncbi.nlm.nih.gov/pmc/articles/PMC4813615/

Prose, N. S., \& Haglund, M. (2015, October 19). How should providers deliver bad news? Retrieved from https://www.youtube.com/watch?v=qHGvjv_7PLU

Rabow, M. W., McPhee, S. J. (1999). Beyond breaking bad news: How to help patients Who suffer. Western Journal of Medicine, 171(4):260-263. 
Reid, M., \& McDowell, J. (2011, August 2). Breaking news of death to relatives. Retrieved from https://www.nursingtimes.net/clinical-archive/end-of-life-andpalliative-care/breaking-news-of-death-to-relatives/5025157.article

Rosenzweig, M., Hravnak, M., Magdic, K., Beach, M., Clifton, M., \& Arnold, R. (2008). Patient communication simulation laboratory for students in an acute care nurse practitioner program [Research Article]. American Journal of Critical Care, 17(4), 364-372.

Rosenzweig, M. Q. (2012). Breaking Bad News: A guide for effective and empathetic communication [Supplemental material]. The Nurse Practitioner, 1-4.

Sangeeta, L., Tyrie, L., Bryczkowski, S., \& Nagurka, R. (2016). Teaching surgery residents the skills to communicate difficult news to patient and family members: A Literature Review. Journal of Palliative Medicine, 19(1), 101-107. doi: https://doi.org/10.1089/jpm.2015.0292

SurveyMonkey Inc. (2018). Retrieved from http://www.surveymonkey.com/

Thomas, A., Stanley, J., Crabtree, M.K., Duffy, E., Dumas, M. A., ... Wolf, A. (2016). Adult-Gerontology Acute Care and Primary Care NP Competencies. Retrieved from http://c.ymcdn.com/sites/www.nonpf.org/resource/resmgr/competencies/NP_Geri _competencies_4.pdf 
W.K. Kellogg Foundation. (2004). W.K. Kellogg Foundation logic model development guide. Retrieved from http://www.wkkf.org/knowledgecenter/resources/2006/02/wk-kellogg-foundation-logic-model-developmentguide.aspx

Wyckoff, M. M., \& Houghton, D. (2009). Critical care: Concepts, role, and practice for the acute and critical care nurse practitioner. New York: Springer.

Venes, D. (Ed.). (2001). Taber's cyclopedic medical dictionary (20th ed.). Philadelphia, PA: F.A. Davis Company

Virshup, B. B., Oppenberg, A. A., \& Coleman, M. M. (1999). Strategic risk management: reducing malpractice claims through more effective patient-doctor communication. American Journal of Medical Quality, 14(4), 153-159. doi:

https://doi.org/10.1177/106286069901400402 


\section{Appendix A}

Pre-Intervention Survey

Control \#

The Impact of a Focused Training on Advanced Practice Nurses'

Experience with Communicating Difficult News

Instructions: This survey is voluntary and confidential. Please abstain from marking this survey with any identifiable markings or text. Circle a number value in the right column that corresponds with the question being asked in the left column. Thank you for your participation.

KEY: $1=$ Strongly Disagree

$2=$ Disagree

$3=$ Undecided

$4=$ Agree

$5=$ Strongly Agree

\begin{tabular}{|c|c|c|c|c|c|}
\hline Survey Questions & $S D$ & $D$ & $U$ & $A$ & $S A$ \\
\hline $\begin{array}{l}\text { I have confidence in my ability to effectively communicate } \\
\text { difficult news to patients. }\end{array}$ & 1 & 2 & 3 & 4 & \\
\hline $\begin{array}{l}\text { I have confidence in my ability to effectively communicate } \\
\text { difficult news to patient families. }\end{array}$ & 1 & 2 & 3 & 4 & 5 \\
\hline $\begin{array}{l}\text { I have a standard method of communicating difficult news to } \\
\text { patients and patient families. }\end{array}$ & 1 & 2 & 3 & 4 & 5 \\
\hline $\begin{array}{l}\text { I am confident in my understanding of the barriers to } \\
\text { communicating difficult news to patients and patient families. }\end{array}$ & 1 & 2 & 3 & 4 & 5 \\
\hline $\begin{array}{l}\text { I have had many opportunities to effectively communicate } \\
\text { difficult news to patients and patient families. }\end{array}$ & 1 & 2 & 3 & 4 & 5 \\
\hline $\begin{array}{l}\text { I am interested in learning an effective method for communicating } \\
\text { difficult news to patients and patient families. }\end{array}$ & 1 & 2 & 3 & 4 & 5 \\
\hline
\end{tabular}




\section{Appendix B}

Post-Intervention Survey

Control \#

The Impact of a Focused Training on Advanced Practice Nurses'

Experience with Communicating Difficult News

Instructions: This survey is voluntary and confidential. Please abstain from marking this survey with any identifiable markings or text. Circle a number value in the right column that corresponds with the question being asked in the left column. Thank you for your participation.

KEY: 1 = Strongly Disagree

$$
\begin{aligned}
& 2=\text { Disagree } \\
& 3=\text { Undecided } \\
& 4=\text { Agree } \\
& 5=\text { Strongly Agree }
\end{aligned}
$$

\begin{tabular}{|c|c|c|c|c|c|}
\hline Survey Questions & SD & $D$ & $\boldsymbol{U}$ & $\boldsymbol{A}$ & $S A$ \\
\hline $\begin{array}{l}\text { I have confidence in my ability to effectively communicate } \\
\text { difficult news to patients. }\end{array}$ & 1 & 2 & 3 & 4 & 5 \\
\hline $\begin{array}{l}\text { I have confidence in my ability to effectively communicate } \\
\text { difficult news to patient families. }\end{array}$ & 1 & 2 & 3 & 4 & 5 \\
\hline $\begin{array}{l}\text { I have a standard method of communicating difficult news to } \\
\text { patients and patient families. }\end{array}$ & 1 & 2 & 3 & 4 & 5 \\
\hline $\begin{array}{l}\text { I am confident in my understanding of the barriers to } \\
\text { communicating difficult news to patients and patient families. }\end{array}$ & 1 & 2 & 3 & 4 & 5 \\
\hline $\begin{array}{l}\text { I have had many opportunities to effectively communicate } \\
\text { difficult news to patients and patient families. }\end{array}$ & 1 & 2 & 3 & 4 & 5 \\
\hline $\begin{array}{l}\text { I am interested in learning an effective method for communicating } \\
\text { difficult news to patients and patient families. }\end{array}$ & 1 & 2 & 3 & 4 & 5 \\
\hline
\end{tabular}

1) I believe this program gave me a better understanding of how to communicate difficult news. YES | NO

2) I believe the skills and knowledge gained during today's' instruction will help me in the future. YES | $\mathrm{NO}$

3) Please include any additional comments in the space provided below. 


\section{Appendix C}

Dear NURS 620 Students,

In approximately 1-week you will be receiving an email with a link to an online pre-test as part of my master's project. You are NOT obligated to complete the pre-test. Not completing the test will in no way be held against you and any and all personal identifying data will not be collected. The pre-test is a short, multiple choice test assessing your comfort level, and experience with the delivery of bad/difficult news in the health care setting. Consent for the pre-test will be implied once you submit your answers. You will all be required to participate in the education session that will occur during NURS 620 skills day on February 8, 2017. Shortly after the completion of skills day, you will receive another email with a link to an online post-test similar to the online pre-test. Again, participation in the post-test is NOT mandatory, and not completing the post-test will in no way be held against you. Please use the next week to submit any questions you have in regard to the pre/post-test.

Enclosed in this e-mail is an attachment to an informational letter explaining in further detail the study I am conducting. Please clink on the attachment and fully read the letter prior to completing

V/R

Nicholas A. Gernt

nagernt@gmail.com 


\section{Appendix D}

Dear NURS 620 Students,

You have taken part in the educational session as part of the NURS 620 skills day. This session addressed teaching points that are based around the SPIKES protocol for the delivery of bad/difficult news. Below is a link to a post-test to be taken after this learning session. This brief test should take no longer than 5-minutes to complete and will be open for 7-days. You are NOT required to take the post-test and choosing not to will in no way be held against you. There will also be no personal data collected during the post-test. Any questions or concerns should be directed to nagernt@gmail.com for further clarification.

$<<<$ SURVEY LINK $>>>$

$\mathrm{V} / \mathrm{R}$

Nicholas A. Gernt

nagernt@gmail.com 


\section{Appendix E}

\section{SPIKES TIPS \& TRICKS}

\section{$\underline{\mathbf{S}}$}

- $\quad$ The "S" refers to the physical setting and the listening skills of the provider

- Establish a quiet and comfortable area for the conversation to take place

- Get your eye on the same level as the patient, and maintain eye contact while listening. *Avoid eye contact when a patient is crying or angry, this may be viewed as an aggressive signal

- Avoid communication across a desk or hard surface, try to sit across from the patient at the corner of a desk. This sends a message that there is a professional/patient interface that you are trying to reach out across and communicate with the patient

- When the patient is speaking, be quiet and listen, DO NOT INTERRUPT

- Use repetition when starting a sentence (e.g. "So what happens now?" "What happens now is..."

$\underline{\mathbf{P}}$

- Before you tell, ask. (e.g. "When you first found the lump in your breast, what did you think of it? Did you think it was serious?")

- Listen to their vocabulary and comprehension, what is their level of understanding? $\underline{\mathbf{I}}$

- The pivotal moment in the interview, if the patient wants information from you, you get a clear invitation to share it.

- $\quad$ Ask the patient their wishes (e.g. "Are you the sort of person who likes the full details about your diagnosis?" "How would you like me to handle the information?"

- Most patients want full information but will remember that you first had the courtesy to ask and all the information they hear from that point on has been at their own request.

$\underline{\mathbf{K}}$

- Start at the level that the patient stopped at, start giving knowledge from the last part of the patients understanding (e.g. "They told me it was a shadow on the lung, nothing serious" "Yes, but you probably don't know that shadows can be caused by many different kinds of things in the lungs")

- Give the information in small chunks, stopping to make sure the patient understands each part (e.g. "Does this make sense to you?")

$\underline{\mathbf{E}}$

- Empathetic response is a technique, not a feeling (e.g. "What I have said must be very upsetting." Or "It must be awful hearing this.")

- Respond to the strongest emotion in the room, if a patient is enraged use a statement such as, "This is making you very angry, obviously"

$\underline{\mathbf{S}}$

- As you are giving feedback, make sure the patient agrees with and understands the plan, ask whether it makes sense or not. Involve the patient in the decision making. 
